\title{
Competencias de la enfermera en instituciones educativas: una mirada desde los gestores educativos
}

\section{Flor Marlene Luna Victoria Mori ${ }^{\mathrm{a}}$ Sebastián Bustamante Edquen ${ }^{b}$ Zoila Esperanza Leitón Espinoza ${ }^{a}$ Rosario Santillán Salazar ${ }^{a}$}

\section{Como citar este artículo:} Luna Victoria Mori FM, Bustamante Edquén S, Leitón Espinoza ZE, Santillán Salazar R. Competencias de la enfermera en instituciones educativas: una mirada desde los gestores educativos. Rev Gaúcha Enferm. 2018;39:e2017-0152. doi: https://doi.org/10.1590/19831447.2018.2017-0152
Universidad Nacional de Truijllo (UNT), Facultad de Enfermería, Departamento de Enfermería del Adulto y Anciano. Trujillo, Peru.

- Universidad Nacional de Trujillo (UNT), Facultad de Enfermería, Departamento de Salud Familiar y Comunitaria. Trujillo, Peru.

\section{RESUMEN}

Objetivo: Describir y analizar los atributos de las competencias de la enfermera en el cuidado de los escolares vista por los gestores de instituciones educativas.

Método: Estudio cualitativo descriptivo-exploratorio. Siete directores de instituciones educativas de Trujillo (Perú) participaron en una entrevista no estructurada, durante el primer semestre del año 2016 y se utilizó el análisis de discurso oral.

Resultados: Categorías empíricas: La enfermera en la promoción de la salud de la comunidad educativa; La enfermera en la prevención de la enfermedad en la comunidad educativa; La enfermera escolar articulando salud-educación en el cotidiano de vida de los escolares.

Consideraciones finales: El estudio contribuye al sustento conceptual de las competencias de la enfermera escolar para la acción intersectorial salud-educación y el establecimiento de políticas de promoción de salud de los escolares dentro las instituciones educativas.

Palabras clave: Salud escolar. Servicios de enfermería escolar. Necesidades y demandas de servicios de salud.

\section{ABSTRACT}

Objective: Describe and analyze the attributes of the nurse competencies in caring of students since the manager look of educational institutions.

Method: Qualitative descriptive-exploratory study. Seven principals of educational institutions of Trujillo (Peru) participates in a non-structured interview during the first semester of 2016 and the analysis of oral discourse was used.

Results: Empirical categories: The nurse in the promotion of educational community health; The nurse in the prevention of the educational community illness; The student nurse articulating health - education in the everyday life of students,

Final considerations: This study contributes to the conceptual support of the competencies of the student nurse for the intersectoral health - education action and the setting-up of health with students in the educational institutions.

Keywords: School health. School nursing. Needs and demands of health services.

\section{RESUMO}

Objetivo: Descrever e analisar os atributos das competências da enfermeira escolar no cuidado escolares a partir da perspectiva dos gestores de instituições de ensino.

Método: Estudo qualitativo descritivo-exploratório: Sete diretores de instituições de ensino em Trujillo (Peru), participaram em uma entrevista no estruturada e se utilizou a análise de depoimentos orais.

Resultados: Os resultados reportam as categorias empíricas: a enfermeira na promoção da saúde da comunidade educativa; a enfermeira na prevenção de doenças da comunidade educativa; A enfermeira escolar articulando a saúde e a educação no cotidiano do escolar.

Considerações finais: 0 estudo contribui ao marco conceitual das competências da enfermeira escolar para a ação inter setorial em saúde-educação e para o estabelecimento de políticas na promoção da saúde dos escolares no interior das instituições educativas. Palavras-chave: Saúde escolar. Serviços de enfermagem escolar. Necessidades e demandas de serviços de saúde. 


\section{口INTRODUCCIÓN}

A nivel mundial las y los adolescentes representan el $20 \%$ de la población total y de esta población, el $85 \%$ se encuentra en los países en vías de desarrollo. En relación a los países de América del Sur, el Perú es considerado como el cuarto país con mayor cantidad de habitantes adolescentes después de Brasil, Colombia y Argentina. Con respecto a América Latina (20 países), el Perú ocupa el quinto lugar, siendo antecedido por Brasil, México, Colombia y Argentina. Se estima que entre 1960 y 1990, el número de adolescentes entre los 10 y 19 años de edad en América Latina aumentó en 138\%; así mismo, en 1999 la población entre los 10 y 24 años de edad alcanzó los 155 millones, representando alrededor de un tercio de la población total de América Latina y El Caribe ${ }^{(1)}$.

Se señala que el desarrollo humano permite que las personas alcancen su máximo potencial de vida productiva y creativa en libertad y con respeto a todo lo que existe en su entorno (2). Lo anteriormente señalado es posible lograrlo cuando se protege a una población en pleno desarrollo como son los escolares y los jóvenes desarrollando sus capacidades y el espacio para hacerlo es dentro de la institución educativa ${ }^{(3)}$. Para conseguir un desarrollo pleno, son claves el acceso a la salud, el bienestar, la educación, la justicia, el empleo y la participación social, además del apoyo a las familias y comunidades para que guíen el desarrollo de los jóvenes. Ello nos lleva a colocar al sector educación en conexión con el sector salud para la promoción de la salud y el desarrollo de adolescentes y jóvenes.

Por otro lado, refieren que del total de adolescentes de 15 a 19 años el $13.9 \%$ ya estuvo alguna vez embarazada. Los mayores porcentajes de maternidad adolescente se presentan entre las mujeres con educación primaria (36.2\%), en las residentes de la Selva (23.7\%), entre las que se encuentran en el quintil inferior de riqueza (23.9\%) y en el área rural (20.3\%). Mientras que menores porcentajes se aprecian en Lima Metropolitana (10.6\%), entre aquellas con educación superior y en las del quintil superior de riqueza ( $8.5 \%$ y $5.6 \%$, respectivamente) ${ }^{(4)}$.

En cuanto a la salud nutricional entre los años 2000 y $2013^{(4)}$ la anemia en las adolescentes mujeres de 15 a 19 años disminuyó de $29 \%$ a $17.7 \%$. Sin embargo, en este aspecto preocupa la creciente aparición del sobrepeso que afecta $23.6 \%$ de adolescentes hombres y mujeres peruanos, y suele ser más frecuente en la zona urbana que en la rural.

En el aspecto educativo se ha incrementado la participación en la escuela secundaria. La cobertura en este nivel de educación llegó a 81.5\% en 2014. Mientras que el porcentaje que culminaba oportunamente la educación se- cundaria, entre los 17 y 18 años de edad, era de $49 \%$ en el 2013, llegó a 64\%.8\% en 2014. Si comparamos los ámbitos urbano y rural existe una brecha importante. Mientras que en el área urbana el $73.2 \%$ de los adolescentes sí termina la secundaria en la edad normativa, sólo $42.3 \%$ lo hace en el medio rural.

Un estudio(5) mostró el nivel de condición física en comparación con valores de referencia nacionales e internacionales, muestran bajos niveles en todas las variables físicas evaluadas. Se observó un 100\% de sobrepeso en todas las edades evaluadas, y sabemos que es en el escolar en quien se va consolidando los estilos de consumo para la obesidad. Del mismo modo la transversalidad en la educación debe incluir la educación en salud como parte de la formación de los jóvenes en la escuela, esto fundamenta aún más y justifica la presencia de profesional de enfermería en las escuelas encargadas de dirigir y promover estilos de vida saludable entre los escolares, siendo uno de ellos, el ejercicio físico ${ }^{(6)}$.

Las escuelas brindan un entorno interesante para promover la salud, pues permiten alcanzar a miles de millones de niños en todo el mundo $y$, a través de ellos, al personal escolar, a las familias y al conjunto de la comunidad(7). Los mensajes de promoción de la salud pueden reforzarse a lo largo de las etapas más determinantes de la vida de los niños, capacitando así a éstos para desarrollar actitudes y aptitudes permanentes. El profesional idóneo para esas acciones es la enfermera dentro de las escuelas públicas, desde una perspectiva del enfoque de complejidad, y bajo estos principios se concibe a la escuela saludable dentro de una organización de trabajo interdisciplinario, intersectorial, y permite tener conocimientos que facilite el desarrollo del ser humano: escolar, niño, adolescente, joven es decir conocer y ejercer ciudadanía(8).

Extrayendo del pensamiento complejo(9) ${ }^{(9)}$ ara esta realidad, diremos que, a nivel general, la interpretación y comprensión de los diversos fenómenos de la naturaleza requieren de una forma de pensamiento distinto, y es donde se destaca el planteamiento relacionado con el desarrollo de un pensamiento de la complejidad en los seres humanos, como una forma de encaminar a los individuos y las naciones hacia el bienestar, la evolución y la productividad. En el desarrollo de un pensamiento de la complejidad en los estudiantes, docentes, está el futuro de la ciencia con conciencia en virtud de los hallazgos que se pueda propiciar.

Brindar servicios de cuidados primarios de enfermería en la promoción de la salud, el bienestar y prevención de problemas de salud de los alumnos; así como, dotar a todas las instituciones educativas del país del profesional en enfermería para garantizar una efectiva protección in- 
tegral de la salud en desarrollo de las garantías y derechos consagrados en el Art $7^{\circ}$ de nuestra Constitución Política. La incorporación del concepto de promoción de la salud escolar dibuja a la escuela promotora de salud, que intenta avanzar en la integración del sector salud y educación donde se ubican; aquí es, donde los gestores educativos tienen un papel importante porque de ellos dependerá que vean o no la necesidad de contar con profesionales enfermeras en la instituciones educativas y que las universidades puedan ser sensibles a las competencias que tienen que formar en la enfermera para el cuidado primario dentro de las Instituciones Educativas. Planteándonos la siguiente interrogante: ¿Qué competencias debe tener la enfermera en las instituciones educativas percibidas por los gestores educativos?, y el objetivo: Describir y analizar los atributos de las competencias de la enfermera en el cuidado de la salud de los escolares vista por los gestores de instituciones educativas.

\section{— METODO}

Es una investigación descriptiva-exploratoria con enfoque cualitativo. El estudio se realizó en la Ciudad de Trujillo, que se localiza en el litoral norte del Perú a una distancia de $500 \mathrm{~km}$. de Lima. El material de estudio fueron las Instituciones Educativas (IEs) del distrito de Trujillo, representadas por sus directores: gestores. El número de participantes de la información fue por saturación según la investigación cualitativa. Todos los datos que se presentan en el estudio fueron obtenidos por entrevista individual no estructurada a siete gestores educativos, con una guía que incluyó las siguientes preguntas abiertas: ¿Qué hace/haría la enfermera dentro de la IE que usted dirige?, ¿Qué otras competencias/ funciones debe tener la enfermera en las IEs?, ¿Qué más puede decir respecto a las enfermeras en las IEs? Los datos obtenidos de la entrevista fueron grabados utilizando una grabadora de voz para tal efecto, y luego transcritos y archivados en un procesador de texto. El recojo de datos fue durante el primer semestre del año 2016. Se señaló como criterio de selección: Institución educativa con población escolar mayor a 500 estudiantes. Gestores con no menos de un año en el cargo. Aceptación y disposición para participar en el trabajo de investigación.

El material que se grabó fue transcrito inmediatamente después de la colecta de datos. Se registró los datos obtenidos por entrevista a los participantes. Se realizó el análisis de discurso oral en la orientación de Orlandi(10), después del recojo de información para luego construir las categorías de las competencias de las enfermeras en las Instituciones Educativas (IEs), previamente se construyó un registro de códigos base. Los códigos reflejan los conceptos centrales presentes en el discurso de los informantes para caracterizar las competencias identificadas por los gestores que la enfermera debe tener en las IEs. Se aseguró el consentimiento informado, anonimato y confidencialidad. La identificación de la pertenencia de las narrativas orales se realiza con letra mayúscula (P) que indica Participante seguida de un número que indica el orden del entrevistado (P1, P2, P3, P4, P5, P6, P7).

\section{ANALISIS Y DISCUSION}

En el presente estudio después de hacer el análisis del material producido por las entrevista, y al contraste con el marco teórico se realizó la categorización en donde se describe las competencias y sus atributos de la enfermera escolar, reportadas como necesarias para la "salud escolar", por los gestores de las instituciones educativas.

La salud no sólo es ausencia de enfermedad, sino todo lo relacionado con el niño y adolescente: su alimentación, juegos, aspiraciones, deseos, y educación; sabemos que, en unos años ellos serán la población económicamente activa del Perú es por ello, que preocupa su formación y desarrollo ya que a futuro serán el soporte de nuestro país.

Después de interpretar, y hacer el análisis de los discursos orales emergen tres categorías empíricas: 1) competencia de la enfermera escolar en la promoción de la salud de la comunidad educativa, 2) competencia de la enfermera escolar en la prevención de la enfermedad de la comunidad educativa, 3) competencia de la enfermera escolar en la articulación de salud-educación en el cotidiano de vida de los escolares.

\section{Competencia de la enfermera escolar en la promoción de la salud de la comunidad educativa}

La inclusión de enfermeras en las instituciones educativas, conllevará a fortalecer el primer nivel de atención con la promoción de la salud en las IEs, y a la vez es el proceso que permite a las personas incrementar el control sobre su salud para mejorarla, abarca no solamente las acciones dirigidas directamente a aumentar las habilidades y capacidades de las personas, sino también las dirigidas a modificar las condiciones sociales y ambientales. Los directores de las instituciones educativas participantes en la entrevista coinciden cuando afirman que:

El tener una enfermera en la escuela nos da seguridad a los profesores y a los padres de familia, aquí tenemos el tópico 
de la enfermera, ella coordina con la psicóloga para que den charlas y fortalezcan la autoestima y madurez emocional. Esto hace que los jóvenes tengan mejor aprovechamiento académico. (P1)

La opción que trabaje una enfermera es muy buena porque "dos cabezas piensan mejor que una" y los alumnos necesitan mejorar, fortalecer su salud, tenemos alumnos que se duermen en clase, otros que no atienden y no entienden, de repente no toman un buen desayuno, o toman desayuno que los hace dormir. Ahora se está trabajando loncheras saludables, cafetines saludables, y la enfermera ayudaría a mejorar las costumbres hábitos de los alumnos, profesores y de los padres de familia. (P6)

Esto evidencia que desde la perspectiva de los gestores educativos la presencia de la enfermera da "seguridad" y sabemos que la presencia de la enfermera es cuidado mismo, ratificado en este discurso, y la enfermera cuida de la autoestima, la madurez emocional, alimentación ${ }^{(11)}$. Es decir, fortalecería en las Instituciones Educativas (IEs) donde aún no hay consultorio de enfermería y fortalece la promoción de la salud, estilos de vida saludable, autoestima, auto concepto, madurez emocional, salud mental, además de la alimentación con loncheras, cafetines saludables, lavado de manos, de dientes, beber agua hervida, ayuda a cambiar, mejorar hábitos, de la comunidad educativa; no solo en los estudiantes sino también en la comunidad educativa: profesores y padres de familia. Porque existe el reconocimiento de parte de los gestores de las instituciones educativas y de los propios padres de familia.

La ampliación o migración de la enfermera hacia el sector educación como espacio de práctica profesional lleva a decir que el éxito para la obtención de la calidad del cuidado enfermero, en este caso del estudio, al escolar, "... está basado en el conocimiento, habilidades, y capacidades de las enfermeras"(12), entendidas como las competencias de la enfermera escolar, que amplían su espacio de actuación desde el sector salud hacia las escuelas. Esto exige un conocimiento de la situación de salud de los escolares, de la competencia que necesita para trabajar en el cuidado primario de enfermería, es decir en el primer nivel de atención.

El Ministerio de Educación desarrolla el Programa para la Promoción de Estilos de Vida Saludables en articulación con el Programa de Alimentación Escolar; entendiendo la promoción de estilos de vida saludable como el desarrollo de habilidades y actitudes de los niños y niñas para que tomen decisiones pertinentes frente a su salud, su creci- miento y su proyecto de vida, y que aporten a su bienestar individual y al colectivo. El programa cumple con el propósito de ofrecer a las entidades territoriales orientaciones conceptuales, pedagógicas y operativas para guiar a los establecimientos educativos en la construcción de proyectos pedagógicos transversales, que contribuyan al desarrollo de conocimientos, habilidades y actitudes en los niños y niñas para que tomen decisiones pertinentes frente a su salud, su crecimiento y su proyecto de vida, y que aporten a su bienestar individual y al colectivo. Sin embargo, se realiza desde desplazamiento extramuros desde el establecimiento de salud hacia las escuelas, que sería diferente si ya la enfermera es trabajadora permanente en el espacio de la escuela. Desde la propia raíz de la enfermería se proclama que la persona, es una realidad a la que hemos de aproximarnos desde una perspectiva holística, sin embargo, la formación de la enfermera ha sido hegemonizada por la búsqueda de formar enfermera con un perfil clínico hospitalario, débilmente incluyendo la promoción de la vida y la prevención en salud, que los discursos antes citados de los directores educativos, reclaman promover la vida, una vida que transcurre entre el hogar y la escuela(13).

Como enfermeras, miembros del equipo de salud, es importante trabajar para cambiar el paradigma de salud. Esto es dar énfasis a lo promocional, preventivo y no a lo recuperativo: "curarse en un hospital". El cambio de paradigma implica entender que el desembolso para salud no es gasto, sino inversión. Si los escolares realizan prácticas saludables: como actividad física, lavado de manos, entre otros; si los escolares tienen un ambiente libre de humo de tabaco, consumo de frutas y verduras, podemos reducir y evitar contraer muchas enfermedades no trasmisibles en las etapas de vida subsiguientes.

Allí importante el papel de enfermería para detectar y actuar en situaciones de sospecha de acoso, abuso sexual o maltrato infantil, para promocionar estilos de vida saludable y sexualidad, en el decir de los gestores [...] fortalecer el autoestima y madurez emocional y por lo tanto mejorar el aprendizaje o el rendimiento académico, sin querer decir que esto responde como "una lógica matemática", si los niños y jóvenes son saludables se tendrá aprendizajes saludables.

La profesión de enfermería desde sus orígenes hasta la actualidad ha ido evolucionando y ocupando espacios no tradicionales, debido, por una parte, a las exigencias de la sociedad y por otra a los avances tecnológicos (tecnología dura y blanda) que hacen que nuestra profesión esté en constante (re)creación, en el presente estudio hablamos, analizamos el espacio de la enfermera en las escuelas. 


\section{Competencia de la enfermera escolar en la prevención de la enfermedad de la comunidad educativa}

La promoción de la salud y la prevención de la enfermedad son dos conceptos inseparables y complementarios; pues la promoción hace referencia al estímulo de los factores protectores para la vida de la población, representados en el fomento de estilos de vida saludables y seguros, nutriendo cuerpo, mente y espíritu; y la prevención está relacionada con el control condiciones y determinantes para no se produzca la enfermedad o esta no se agrave, tal como manifiestan los directores durante la entrevista:

Los alumnos empiezan a fumar, aunque está prohibido y ellos lo saben, vienen con la ropa impregnada de cigarrillos, el consumo de alcohol empezó en algunos alumnos a los 10, 12 años, es preocupante, porque en algunos casos no tenemos el apoyo de sus padres. (P3)

Se sabe que la prevención es la meta fundamental y más deseable para todas las enfermedades, pero en el caso de las adicciones se presenta un reto complejo, por el número y variedad de sustancias, sus efectos en los individuos, el contexto social y legal en que se encuentran.

El profesional de enfermería, no sólo realiza actos sino que también muestra "actitud de cuidado"(14), ejercerá liderazgo, visibilizando el hacer y quehacer en el espacio del cuidado del niño y adolescente, sano y con problemas de salud agudos, crónicos. La enfermera está capacitada en las competencias de planificar, ejecutar y evaluar programas de salud, programas sociales, realizar investigaciones, educación de acuerdo a cada realidad.

En el proyecto de ley ${ }^{(15)}$ que se encuentra en el Congreso de la República presentado por el Colegio de Enfermeros del Perú (CEP) para su evaluación y discusión, que incorpora al profesional en enfermería en todas las instituciones educativas a nivel nacional en el Perú, en su artículo tres de los objetivos, puntualiza que "las normas y procedimientos contemplados en esta ley ordenarán las competencias de la enfermera escolar de las diferentes entidades educativas que prestan servicios en materia de salud escolar" para lograr objetivos como: detección precoz de enfermedades no comunicables: obesidad, hipertensión, diabetes, entre otras. Y la educación contra la violencia escolar/familiar, consumo de drogas, embarazo precoz y educación en salud reproductiva. Este es un rol fundamental de las enfermeras/os en las escuelas tal como lo manifiestan en la entrevista:
El problema del uso de drogas es complejo, a veces el alumno se duerme en clases, el rendimiento es bajo, están apáticos, no cumplen con las reglas de la institución. En esos casos llamamos al padre/madre de familia, o apoderado para que tome cartas en el asunto. iMuchas veces los padres se niegan a ver la realidad, y los profesores con la tutoría no pueden hacer mucho, porque los casos son delicados, nosotros sugerimos algunas cosas nada más! jComo que lo lleven al médico, o que investiguen quienes son sus amigos. (P4)

La presencia de la enfermera facilitará el camino para la atención de estos casos de carácter preventivo de la salud del escolar y para la familia para disminuir la vulnerabilidad y ejercer un impacto sobre la calidad de vida de los escolares y adolescentes y sus familias, y si la enfermera está en las escuelas, se encargará de ejercer el cuidado de la salud al público de la comunidad escolar: alumno/a-padre/madre de familia-maestro/a.

La enfermera en las escuelas con pertinencia realiza el trabajo de prevención imbuida en un trabajo sostenible y de salud pública, como lo dicen los directores:

Es importante hacer un trabajo coordinado, dar charlas para prevenir enfermedades, para curarlas. Son pocos, pero si hemos tenido alumnos con cáncer, cuando nuestros estudiantes se enferman ya no vienen al centro o los padres o madres de familia resultan con esa enfermedad y tenemos abandono o irregularidad en la asistencia. (P7)

La ausencia de los alumnos se ve incrementada por la presencia de enfermedades frecuentes y poco frecuentes, las cuales son significativas por la ausencia escolar que generan. Estas enfermedades pueden ser no transmisibles - o crónicas - siendo estas afecciones de larga duración con una progresión generalmente lenta. Se resalta la necesidad de trabajar en la prevención de enfermedades transmisibles y no trasmisibles, tomando en cuenta la responsabilidad de cada uno de nosotros sobre el cuidado de nuestra salud y la de nuestra familia.

También se evidencia que la presencia de la enfermera da seguridad como se expresa en los siguientes discursos:

La enfermera atiende inmediatamente a los alumnos, si pasa cualquier cosa: tienen algún dolor de cabeza, dolor de estómago, resfrío, bronquios, crisis asmática, está la enfermera para que los atienda, eso da mucha seguridad. Cuando pienso en una enfermera en la escuela, me imagino que sus tareas tienen que ver con las vacunas, la atención en caso de accidentes escolares, cuidado de la salud... entre otras tareas. (P5) 
Para fijar los conocimientos, habilidades es en la infancia, y a lo largo de los años se tiene que trabajar de forma continua con los niños, adolescente, con el fin de adquirir hábitos saludables que influirán positivamente en la salud y la vida. La educación para la salud, tendría que ser un curso aparte, iqué se debe dictar se debe!, como educación física, educación para la salud física, mental, hay muchos problemas ahora con los niños, la crianza ha cambiado, y por lo tanto también ha cambiado los comportamientos. (P2)

"[...]"A veces hay apoyo de los padres otras veces no, creen que la escuela les dará todo, pero sabemos que no es así, si los padres no colaboran en la educación, formación de sus hijos. Casi nada o muy poco hacemos los profesores. (P3)

La enfermera en el espacio de las escuelas, integrada en la Comunidad Educativa, es un valor agregado, de calidad que enlazados con el equipo de profesores trabaja para normalizar la vida diaria del niño y adolescente, sano o con problemas de salud, fomentando hábitos de vida saludables en toda la población escolar y comunidad educativa, tal como está planteado en el proyecto de ley, que incorpora al profesional en enfermería en todas las instituciones educativas a nivel nacional en su artículo cuarto sobre Designación de un profesional de Enfermería, "declárese la necesidad de designar por lo menos un profesional de Enfermería en cada institución educativa encargado de la prestación de los servicios de cuidados primarios de enfermería en la promoción y prevención de problemas de salud de los estudiantes "[...]" y en su artículo quinto referente al Ámbito de Aplicación refiere "[...]" el conjunto de instituciones educativas públicas del nivel básico (primario y secundario) y privadas, en materia de la enfermería escolar se ordenará en función de la realización de un trabajo en red, a través del que se garantice la prestación integral de servicios en salud escolar y se generen procesos participativos de optimización de la calidad en la prestación de servicios para el escolar y adolescente" ${ }^{\prime \prime(15)}$.

Como vemos y como decíamos anteriormente, la presencia de la enfermera en las escuelas se convierte en una necesidad social, de salud y la presencia física de la enfermera es cuidado, tal como lo manifestaron los directores. Se reporta que $\mathrm{e}^{(16-17)}$ en el momento de cuidar a la persona, es esencial la presencia física, el cuerpo como espacio mínimo de subjetividades y objetividades de la enfermera establece relaciones de cuidado, con otros espacios, con otros cuerpos, que se funden y entraman en la cotidianidad, es en el presente trabajo que el cuerpo enfermera interrelaciona con el cuerpo alumno y comunidad escolar.

\section{Competencia de la enfermera escolar en la articulación de salud-educación en el cotidiano de vida de los escolares.}

Dentro de las competencias de la enfermera escolar está la articulación de la educación para la salud en donde se conjuga el aprehender académico escolar y el aprender para la vida, salud, bienestar, en el contexto de los programas de salud escolar, ejecutados por las profesionales de la salud, ya sea desde la proyección de los centros de salud, o desde los consultorios de enfermería escolar, enfatizando en la promoción de la salud, con los estilos de vida saludable, vacunación, crecimiento y desarrollo, atención de adolescentes, detección temprana de los problemas más frecuentes, especialmente aquellos que se relacionan con la asistencia y el rendimiento escolar. Los directores educativos afirman que:

La educación y la salud, son los pilares fundamentales del desarrollo de la sociedad, si los niños tienen salud, buena salud van a rendir más, si los niños tienen educación serán profesionales, empresarios, empleados y tendrán su trabajo, ganarán dinero y tendrán buena salud y serán hombres y mujeres de bien. Las enfermeras ayudarían a mantener la salud y mejorar la vida. (P1)

Es muy importante la educación en salud a edad temprana, lo que el niño aprende y lo que practica no lo olvida, además que lo que dice su maestra lo cumplen al pie de la letra, cuanto más chiquitos mejor, si lo dice la enfermera también porque la van a ver como su maestra. (P3)

En el cotidiano de vida de los escolares, la salud-educación se articulan en las políticas de escuelas saludables, para el mejoramiento del ambiente escolar, con la participación activa de los alumnos, los profesores, familias y comunidad, para reforzar la ciencia con conciencia del vínculo que integra la salud con educación y la educación con salud de quienes son responsables del proceso pedagógico de enseñar-aprender y vivir en la escuela. Por ejemplo en la obesidad, únicamente la Secretaría de Salud y la Secretaría de Educación acordaron restringir la venta de alimentos y bebidas en las escuelas ${ }^{(18)}$.

Sería importante, que además de la mirada desde la salud y la educación estuvieran también presentes las aportaciones de las neurociencias, la endocrinología, la nutriología, la pedagogía, la sociología, la antropología y la dirección de sistemas de salud, entre otras; en suma, la intersectorialidad es importante. Lo antes referido, nos invita a reflexionar nuevamente sobre las necesidades y oportu- 
nidades que caracterizan el escenario de la promoción de salud, prevención de enfermedades y articulación de estos dos pilares fundamentales para el desarrollo social, cultural, académico en el ámbito escolar. A continuación, se relatan los discursos de los directores que coinciden cuando dicen:

Las enfermeras también tienen función educativa, porque ellas dan charlas, educan para tener buenos hábitos de higiene, a toda la comunidad educativa, padres, profesores, alumnos. También sería interesante e importante para la institución hacer investigaciones, las enfermeras investigan un poco más que las profesoras y de repente allí también se motivan y hacen investigaciones que a nosotros como institución nos interesa. (P4)

Se requiere al personal de salud: a la enfermera y que pueda coordinar con el profesor para que atienda los casos particulares de cada alumno. (P6)

Se evidencia en el trabajo de la enfermera su rol de educadora, como lo manifestado por los directores, el concepto de intersectorialidad va de la mano con la integración, en donde los sectores trabajan juntos y tienen que constituir nexos de integración, la presencia de la enfermera en las escuelas, la torna en interlocutora para el sector salud pero desde el espacio del sector educación ${ }^{(19)}$.

La correcta implantación de un sistema de gestión por competencias, permite: la descripción de puestos; la integración de equipos de trabajo; la implantación de una cultura organizativa; la reducción de la barrera generacional; la apreciación del potencial; la dirección por objetivos; y la gestión del cambio.

La propuesta que trabaje una enfermera es muy buena porque se daría "integración" Los centros educativos tenemos una debilidad, mejor dicho, el sector educación es olvidado, falta presupuesto, los profesores ganamos poco, falta perfil institucional, la concepción de trabajo interdisciplinario es escaso, falta creatividad, la mayoría solo esperamos directivas y cumplirlas. (P2)

En verdad que en las escuelas tendría que haber profesores, enfermeras, psicólogos, nutricionistas. Tener un consultorio de salud en cada escuela, imagínese con eso los alumnos tendrían salud física, emocional, mental, ambiental, social. (P3)

De lo dicho por los gestores, se afirma que la enfermera escolar es interlocutora y forma parte del sector educación y es el enlace con el sector salud, le imprime a la enfermería un nuevo atributo de identidad(20) definida como "el conjunto relacionado de atributos culturales, al que se da prioridad sobre el resto de las fuentes de sentido". Aclara que no debe confundirse la identidad con los roles sociales, definimos como las normas estructuradas por instituciones y organizaciones de la sociedad, en este caso de estudio por las escuelas. La identidad se construye mediante un proceso de individualización y autodefinición. "[...]"Las identidades organizan el sentido, los roles organizan las funciones", que hace diferente a enfermería al cubrir con su práctica profesional nuevos espacios de actuación.

\section{口CONSIDERACIONES FINALES}

La enfermera escolar dentro de sus competencias en la promoción de la salud de la comunidad educativa; plantea las siguientes hipótesis de trabajo para el meta-análisis realizado y la propuesta de competencias de la enfermera escolar: La salud escolar, ayudará a desarrollar una cultura de salud, bienestar y ayudará en la modificación de las condiciones sociales, ambientales en la comunidad educativa, especialmente en los alumnos: niños, adolescentes, padres/madres de familia, profesores. La presencia de la enfermera se expresa en el cuidado, fortalece estilos de vida saludable, autoestima, auto concepto, madurez emocional, salud mental, además de la alimentación con loncheras, cafetines saludables, lavado de manos, de dientes, beber agua hervida, ayuda a cambiar, mejorar hábitos, de la comunidad educativa. La enfermera escolar fortalecerá el rol protagónico de los padres de familia porque los hábitos, creencias, costumbres en la alimentación (in) saludable es una práctica que comienza en casa y en familia.

Se observa en las escuelas, en donde hay una enfermera, en el consultorio de enfermería un impacto en la articulación interdisciplinaria: escuelas saludables aprendizajes saludables. Competencias de la enfermera escolar en la prevención de la enfermedad de la comunidad educativa. La enfermera escolar, es el profesional capacitado para proporcionar cuidados de enfermería especializados de forma autónoma, en el ámbito educativo en un equipo interdisciplinario e intersectorial, por tanto, un papel intersectorial, nuevos atributos de identidad de ser enfermera. La presencia de la enfermera en las escuelas se convierte en una necesidad social, de salud y la presencia física de la enfermera es el cuidado.

La enfermera escolar realiza articulación, integración en el espacio de vida de los escolares, espacio privilegiado de educación, espacios de aprendizaje para la salud, para la vida. La enfermera escolar cuida a la población de la comunidad educativa, y se convierte en enfermera perenne. Su 
práctica requiere de un pensamiento interdisciplinario, intersectorial, en equipo, con visión de integración, singularidad, diversidad, colectividad en su relación con la familia, sociedad y ambiente.

Lo antes mencionado permitirá que las facultades de enfermería de las universidades incluyan en los programas curriculares a la salud escolar como un trabajo en el sector educación y no como tradicionalmente se ha entendido que la salud escolar que es una extensión del sector salud al sector educación. En el área asistencial, que la enfermera puede ejercer su competencia de la enfermera escolar como parte del personal del sector educación dirigido a los docentes, escolares, gestores y padres de familia. Estudio también es una base empírica para dar base al proyecto de ley de incorporar una enfermera en cada escuela pública y privada para fortalecer el primer nivel de atención y la ampliación de los espacios de práctica profesional de la enfermera. El estudio explora la posibilidad de realizar investigaciones que responda a la búsqueda de nuevas dimensiones organizacionales salud-educación tanto en lo público y privado, para operacionalizar el trabajo de enfermería en el primer nivel de atención en una acción interdisciplinaria e intersectorial salud-educación para el desarrollo, bienestar, felicidad de los escolares peruanos. Finalmente, la limitación de este estudio es su abordaje exploratorio en una localidad mus circunscrita a una localidad del litoral peruano y que sólo se haya incluido a gestores de las IEs.

\section{REFERENCIAS}

1. Ministerio de Salud (PE). Análisis de situación de salud de las y los adolescentes. Ubicándolos y Ubicándonos [Internet]. Lima: Ministerio de Salud; 2012 [citado 2017 oct 08]. Disponible en:https://www.mimp.gob.pe/files/planes/Plan_Nacional_PNAIA_2012_2021.pdf.

2. Ministerio de la Mujer y Poblaciones Vulnerables (PE). Plan nacional de acción por la infancia y la adolescencia 2012-2021. Lima: Editores Impresores; 2012 [citado 2017 oct 08]. Disponible en:https://www.mimp.gob.pe/files/planes/ Plan_Nacional_PNAIA_2012_2021.pdf.

3. OCDE/CEPAL/CAF. Perspectivas económicas de América Latina 2017: juventud, competencias y emprendimiento. 2016. Paris: Éditions OECD; 2016. doi: http:// dx.doi.org/10.1787/leo-2017-es.

4. Ministerio de Salud (PE). Documento Técnico Análisis de situación de salud de las y los adolescentes: ubicándolos y ubicándonos. Lima: El Ministério; 2009 [citado 2017 mar 01]. Disponible en: ftp://ftp2.minsa.gob.pe/normaslegales/2009/RM636-2009.pdf.

\section{Autor correspondente:}

Flor Marlene Luna Victoria Mori E-mail:flor_mlvm@yahoo.com
5. Hernández-Mosqueira C, Fernandes da Silva S, Ibarra Mora J, Hernández Vasquez D, Caniuqueo Vargas A, Esparza Muñoz E, et al. Nivel de condición física orientada a la salud en estudiantes varones de 10 a 14 años de Colegio Darío Salas Chillan. Mot Hum. 2015 [citado 2017 oct 11];16(1):33-40. Disponible en:https://www.researchgate.net/publication/282132797_nivel_de_condicion_fisica_orientada_a_la_salud_en_estudiantes_varones_ de_10_a_14_anos_del_colegio_dario_salas_chillan.

6. Aguirre FM. Transversalidad y educación para la salud: prevención de drogas en secundaria [Tesis Maestría]. Almería: Universidad de Almería; 2012.

7. Maurer FA, Smith CM. Community/public health nursing practice: health for families and populations. 5th ed. St. Louis: Elsevier/Saunders; 2013.

8. Weber M. Ensayos de sociología contemporánea. Tra ed. Barcelona: PlanetaAgostini; 1985.

9. Morín E. Los siete saberes necesarios para la educación del futuro. 1ra ed. Paris: Unesco; 1999.

10. Orlandi E. Análisis de discurso: principios y procedimientos. 1ra ed. Santiago: Lom; 2012.

11. Waldow VR. Bases e principios do conhecimiento e da arte da enfermagem. 1ra ed. Rio de Janeiro: Vozes; 2008.

12. Kérouac S, Pepin J, Ducharme F. El pensamiento enfermero. 1ra ed. Barcelona: Masson; 2010.

13. Bardallo PD. Entre la enseñanza y el aprendizaje: un espacio de saberes para compartir. [Tesis Doctoral]. Barcelona: Universitat de Barcelona; 2010.

14. Silva LWS, Francioni FF, Sena ELS, Carraro TE, Randünz V. 0 cuidado na perspectiva de Leonardo Boff, uma personalidade a ser (re)descoberta na enfermagem. Rev Bras Enferm. 2005 ago[citado 2017 oct 11]; 58(4):471-5. Disponible en: http://www.scielo.br/scielo.php?script=sci_ arttext\&pid=S0034-71672005000400018\&lng=en.

15. Proyecto de Ley Número 01706/2016-CR presentado al Congreso de la República. Perú: Congreso de la República del Perú; 2016 [citado 2017 oct 08]. Disponible en: http://www.leyes.congreso.gob.pe/Documentos/2016_2021/ Proyectos_de_Ley_y_de_Resoluciones_Legislativas/PL0170620170725.pdf.

16. Vinalay C, Luna Victoria FM. Resonancias y disonancias en el proceso de cuidar durante el puerperio. Desarrollo CientifEnferm. 2012[citado 2017 oct08];20(9):2915. Disponible en: http://www.index-f.com/dce/20pdf/20-291.pdf.

17. Sosa Rosas M, Luna Victoria MF. La corporalidad de la persona con cáncer. Rev Conamed. 2013[citado 2017 oct 08];18(Supl 1):S17-S23. Disponible en: https://dialnet.unirioja.es/descarga/articulo/4701488.pdf.

18. Urbina Fuentes M, González Block M. La importancia de los determinantes sociales de la salud en las políticas públicas. 1ra ed. Cuernavaca: Instituto Nacional de Salud Pública (Mx); 2012 [citado 10 oct 2017]. Disponible en: http://medicinaweb.cloudapp.net/observatorio/docs/ss/i/Ss2012_Li_Importancia.pdf.

19. Cunill-Grau N. La intersectorialidad en las nuevas políticas sociales: un acercamiento analítico-conceptual. Gest Polít Pública. 2014[citado 2017 oct 10]; 23(1):5-46. Disponible en:http://www.scielo.org.mx/scielo.php?script=sci_ arttext\&pid=\$1405-10792014000100001\&lng=es\&nrm=iso.

20. Castells M. El poder de la identidad en la era de la información. México: Alianza Editorial; 2003. v. 2

Recebido: 05.08.2017

Aprovado: 28.11.2017 